\title{
Orientações para o cuidado domiciliar de crianças e adolescentes em quimioterapia antineoplásica oral
}

\author{
Guidance for homecare of children and teenagers in oral antineoplastic chemotherapy \\ Guias para el cuidado en el hogar de niños y adolescentes en quimioterapia antineoplástica oral
}

Recebido: 31/03/2021 | Revisado: 05/04/2021 | Aceito: 12/04/2021 | Publicado: 16/04/2021

\author{
Gabriele Alvernaz Silva Franco \\ ORCID: https://orcid.org/0000-0002-5456-531X \\ Instituto Nacional do Câncer, Brasil \\ E-mail: gabrielealvernaz@yahoo.com.br \\ Liliane Faria da Silva \\ ORCID: https://orcid.org/0000-0002-9125-1053 \\ Universidade Federal Fluminense, Brasil \\ E-mail: lili.05@ hotmail.com \\ Flavio Luiz Seixas \\ ORCID: https://orcid.org/0000-0002-7160-0818 \\ Universidade Federal Fluminense, Brasil \\ E-mail: fseixas@ic.uff.br \\ Fernanda Garcia Bezerra Góes \\ ORCID: https://orcid.org/0000-0003-3894-3998 \\ Universidade Federal Fluminense, Brasil \\ E-mail: ferbezerra@gmail.com \\ Emília Gallindo Cursino \\ ORCID: https://orcid.org/0000-0002-5845-9709 \\ Universidade Federal Fluminense, Brasil \\ E-mail: egcursino@globo.com
}

\begin{abstract}
Resumo
Objetivos: Identificar os quimioterápicos antineoplásicos orais mais utilizados pelas crianças e adolescentes em tratamento ambulatorial; e descrever, a partir da literatura científica, as principais orientações para os cuidados domiciliares às crianças e adolescentes que usam quimioterápicos antineoplásicos orais. Método: Estudo descritivo, realizado em um hospital federal na cidade do Rio de Janeiro, Brasil. Foram analisados os prontuários de 45 crianças e adolescentes em tratamento com quimioterápicos orais, no período de julho a setembro de 2020. Resultados: Os quimioterápicos utilizados foram: mercaptopurina $(29,1 \%)$, metrotexato $(21 \%)$, imatinibe $(19,3 \%)$, temozolamida $(9,7 \%)$, everolimus $(8,1 \%)$, ciclofosfamida $(6,4 \%)$, tioguanina $(1,6 \%)$, etoposideo $(1,6 \%)$, topotecano $(1,6 \%)$ e pazopanibe $(1,6 \%)$. Assim, para cada quimioterápico, foram elencadas as principais orientações para os cuidados domiciliares às crianças e adolescentes em uso deles. Considerações finais: $\mathrm{O}$ estudo possibilita direcionamento das ações de orientação para o cuidado domiciliar de crianças e adolescentes em quimioterapia antineoplásica oral visando à adesão e maior segurança no tratamento.
\end{abstract}

Palavras-chave: Criança; Adolescente; Antineoplásicos; Administração Oral.

\begin{abstract}
Objectives: identify the oral antineoplastic chemotherapy most used by children and teenagers in outpatient treatment; and describe the guidance for homecare of children and teenagers that use antineoplastic chemotherapy using scientific literature as main source. Method: A descriptive study was performed in a federal hospital in the city of Rio de Janeiro, Brazil. The medical records of 45 children and teenagers in treatment using oral chemotherapy were analyzed from July to September of 2020. Results: The chemoteherapy used was mercaptopurine (29,1\%), methotrexate $(21 \%)$, imatinib $(19,3 \%)$, temozolamide $(9,7 \%)$, everolimus $(8,1 \%)$, cyclophosphamide $(6,4 \%)$, thioguanine $(1,6 \%)$, etoposide $(1,6 \%)$, topotecan $(1,6 \%)$ and pazopanibe $(1,6 \%)$. For each substance part of the chemotherapy the main guidance was listed for homecare of children and teenagers. Final considerations: This study allows the targeting of the guidance actions for homecare of children and teenagers in oral antineoplastic chemotherapy aiming to its accession and a higher safety in treatment.
\end{abstract}

Keywords: Child; Adolescent; Antineoplastic Agents; Administration, Oral.

\section{Resumen}

Objetivos: Identificar quimioterápicos antineoplásicos orales más utilizados por los niños y adolescentes en tratamiento ambulatorio; y describir, a partir de la literatura científica, las principales orientaciones para los cuidados domiciliares para los niños y adolescentes que utilizan quimioterapia antineoplásica oral. Método: un estudio 
descriptivo fue realizado en un hospital federal en la ciudad de Rio de Janeiro, Brasil. Los registros médicos de 45 niños y adolescentes en tratamiento quimioterápicos antineoplásicos orales fueran analizados en el período entre Julio y Septiembre de 2020. Resultados: los quimioterápicos utilizados fueran: mercaptopurina $(29,1 \%)$, metrotexato (21\%), imatinib $(19,3 \%)$, temozolamida $(9,7 \%)$, everolimus $(8,1 \%)$, ciclofosfamida $(6,4 \%)$, tioguanina $(1,6 \%)$, etopósido $(1,6 \%)$, topotecan $(1,6 \%)$ y pazopanibe $(1,6 \%)$. Entonces, para cada quimioterapia, fueran listados las principales guías para los cuidados en el hogar de niños y adolescentes en su uso. Consideraciones finales: el estudio permite dirigir las acciones de guía para el cuidado en el hogar de niños y adolescentes en quimioterapia antineoplásica oral apuntando a la adherencia y seguridad en el tratamiento.

Palabras clave: Niño; Adolescente; Antineoplásicos; Administración Oral.

\section{Introdução}

O câncer infantojuvenil representa a primeira causa de morte por doenças crônicas entre as crianças e adolescentes no Brasil. Estima-se que ocorrerá, em cada ano do triênio 2020-2022, cerca de 4.310 novos casos de câncer infantojuvenil no sexo masculino e de 4.150 do sexo feminino, sendo, os tipos predominantes, as leucemias (28\%), os tumores do sistema nervoso central (26\%) e os linfomas (8\%) (INCA, 2020a).

O câncer infantojuvenil não pode ser considerado uma simples doença, pois corresponde a um grupo de várias doenças que têm em comum a proliferação descontrolada de células anormais com características próprias em relação à histopatologia, comportamento clínico, localização primária do tumor, etnia, sexo e idade (INCA, 2020a; Iuchno \& Carvalho, 2020).

Os diversos tipos de câncer são tratados associando-se ou não, de acordo com o caso, cinco modalidades terapêuticas: cirurgia, quimioterapia, radioterapia, transplante de células-tronco hematopoiéticas e terapia biológica. Neste estudo, destacamse as drogas quimioterápicas que, no tratamento de crianças e adolescentes com câncer, contribuíram de maneira significativa para as taxas de sobrevida, já que os tumores pediátricos têm alto índice proliferativo e são, na sua maioria, quimiossensíveis (Iuchno \& Carvalho, 2020).

A principal definição de quimioterapia antineoplásica refere-se ao emprego de substâncias químicas, isoladas ou em combinação, que atuam interferindo diretamente no processo de crescimento e divisão do ciclo celular com o objetivo de tratar as neoplasias malignas. Os protocolos de quimioterapia podem ser compostos de drogas de administração venosa, oral, intratecal, intramuscular, subcutânea, intracavitária e intra-arterial (Bonassa \& Gato, 2012). Seu uso terapêutico pode causar vários efeitos adversos e toxicidades, sendo sua atuação inespecífica, ocorrendo de forma sistêmica em células de rápida proliferação e não apenas em células neoplásicas. Os efeitos adversos e as toxicidades mais frequentes são: hematológicas, infecciosas, gastrointestinais, urológicas, neurológicas, pulmonares, disfunções cardíacas e reações alérgicas (Iuchno \& Carvalho, 2020; Bonassa \& Gato, 2012).

Durante muitos anos, utilizou-se exclusivamente a terapia antineoplásica intravenosa, mas o panorama do tratamento do câncer vem mudando com o uso expansivo da terapia antineoplásica oral, tornando-se um componente importante no tratamento das leucemias, tumores do sistema nervoso cerebral e para os tumores sólidos depois que a quimioterapia intravenosa deixa de ser eficaz (Mesquita \& Silva, 2016; Marshall \& Cairns, 2019).

O uso dos antineoplásicos orais no domić́lio exige que familiares e cuidadores enfrentem novos desafios, pois estes se tornam responsáveis pela administração, autogerenciamento dos sintomas e dos efeitos colaterais e pela adesão ao tratamento (Marshall \& Cairns, 2019).

Os profissionais de saúde, principalmente os enfermeiros, pela proximidade com a família durante o tratamento, devem estar preparados para orientar de forma humanizada e realizar as intervenções assistenciais e educativas necessárias, de modo a auxiliar os familiares no cuidado aos seus filhos em tratamento com os antineoplásicos orais (Fernandes, Silva, Tacla, Ferrari \& Gabani, 2018). 
Para melhor entender esse contexto, foram levantadas as seguintes questões norteadoras: Quais os quimioterápicos antineoplásicos orais mais utilizados pelas crianças e adolescentes em tratamento ambulatorial? Quais são as principais recomendações que devem ser realizadas durante a consulta de enfermagem para os cuidados domiciliares às crianças e adolescentes que usam quimioterápicos antineoplásicos orais?

Assim, os objetivos foram: Identificar os quimioterápicos antineoplásicos orais mais utilizados pelas crianças e adolescentes em tratamento ambulatorial; e descrever, a partir da literatura científica, as principais orientações para os cuidados domiciliares às crianças e adolescentes que usam quimioterápicos antineoplásicos orais.

\section{Metodologia}

O presente estudo descritivo é um recorte de uma pesquisa de mestrado em enfermagem. O cenário foi um hospital federal, referência no ensino, pesquisa e tratamento do câncer, localizado na cidade do Rio de Janeiro. Este hospital possui duas centrais de quimioterapia, uma destinada ao atendimento do público adulto e a outra, ao atendimento de pacientes infantis.

O fluxo de atendimento médio do ambulatório da quimioterapia infantil é de 25 crianças por dia. Este setor funciona de 07 horas às 19 horas, em todos os dias da semana, incluindo feriados. Para o atendimento, possui duas enfermeiras plantonistas e uma diarista; e o enfermeiro da quimioterapia infantil também é o responsável por administrar o quimioterápico dos pacientes internados na pediatria e hematologia infantil e no Centro de Terapia Intensiva (CTI) infantil.

Para a seleção da amostra, foi solicitada ao serviço de farmácia a listagem das crianças e adolescentes cadastrados em tratamento com quimioterápicos orais. Assim, foram incluídos no estudo as 45 crianças e adolescentes que estavam em tratamento com quimioterápicos orais, no período de julho a setembro de 2020.

Para a operacionalização dessa etapa, utilizou-se uma planilha do Excel, garantindo a padronização e a sequência na coleta dos dados. A partir da leitura dos 45 prontuários eletrônicos, foi possível realizar o levantamento das seguintes variáveis: sexo, idade, tempo do diagnóstico, diagnóstico, quimioterápico oral prescrito e quantidade de quimioterápicos diferentes prescritos. Os dados coletados foram analisados por meio de análise descritiva e apresentados por porcentagens.

Após a identificação dos quimioterápicos antineoplásicos orais mais utilizados pelas crianças e adolescentes em tratamento ambulatorial, foi realizada uma busca na literatura científica nacional e internacional a fim de identificar evidências para que pudessem ser elencadas as principais orientações aos cuidados domiciliares a essas crianças e adolescentes.

Os preceitos éticos que envolvem as pesquisas com seres humanos foram respeitados e a coleta de dados ocorreu após a aprovação pelo Comitê de Ética em Pesquisa da instituição proponente (Parecer 4.012.929, CAAE: 30134320.2.0000.524) e da instituição coparticipante onde a pesquisa foi realizada (Parecer 4.074.187, CAAE: 30134320.2.3001.5274). Todos os dados foram manejados e analisados de forma anônima.

\section{Resultados e Discussão}

Foram identificadas 45 crianças e adolescentes (Tabela 1), que constituíram a amostra do estudo, no período de julho a setembro de 2020, em tratamento com quimioterápicos orais; as informações foram extraídas dos prontuários eletrônicos. 
Tabela 1. Características das crianças e adolescentes em tratamento com quimioterápicos orais. Rio de Janeiro, RJ (2020).

\begin{tabular}{|c|c|c|}
\hline Variável & $n=(45)$ & $\mathbf{f}(\%)$ \\
\hline \multicolumn{3}{|l|}{ Sexo } \\
\hline Feminino & 19 & 42,3 \\
\hline Masculino & 26 & 57,7 \\
\hline \multicolumn{3}{|l|}{ Idade } \\
\hline Lactente & 0 & 0 \\
\hline Pré-escolar & 4 & 8,9 \\
\hline Escolar & 17 & 37,8 \\
\hline Adolescente & 24 & 53,3 \\
\hline \multicolumn{3}{|l|}{ Tempo do diagnóstico } \\
\hline Meses $\vdash 2$ anos & 15 & 33,4 \\
\hline $2 \longmapsto 4$ anos & 17 & 37,8 \\
\hline $4 \vdash 6$ anos & 5 & 11,1 \\
\hline $6 \vdash 8$ anos & 2 & 4,4 \\
\hline mais de 8 anos & 6 & 13,3 \\
\hline \multicolumn{3}{|l|}{ Diagnóstico } \\
\hline Leucemia Linfocítica Aguda (LLA) & 17 & 37,8 \\
\hline Leucemia Mieloide Crônica & 5 & 11,2 \\
\hline Linfoma Linfoblástico B & 2 & 4,4 \\
\hline Linfoma Linfoblástico T & 1 & 2,2 \\
\hline Histiocitose de Células de Langerhans & 3 & 6,7 \\
\hline Meduloblastoma & 2 & 4,4 \\
\hline Glioma difuso de alto grau & 2 & 4,4 \\
\hline Esclerose Tuberosa & 5 & 11,2 \\
\hline Ependimoma & 1 & 2,2 \\
\hline Teratoide rabdoide atípico de SNC & 1 & 2,2 \\
\hline Rabdomiossarcoma & 3 & 6,7 \\
\hline Osteossarcoma & 2 & 4,4 \\
\hline Tumor desmoplásico abdominal & 1 & 2,2 \\
\hline \multicolumn{3}{|l|}{ Quimioterápico oral } \\
\hline Mercaptopurina & 18 & 29,1 \\
\hline Metotrexato & 13 & 21 \\
\hline Imatinibe & 12 & 19,3 \\
\hline Temozolamida & 6 & 9,7 \\
\hline Evorolimos & 5 & 8,1 \\
\hline Ciclofosfamida & 4 & 6,4 \\
\hline Tioguanina & 1 & 1,6 \\
\hline Etoposideo & 1 & 1,6 \\
\hline Topotecano & 1 & 1,6 \\
\hline Pazopanibe & 1 & 1,6 \\
\hline \multicolumn{3}{|l|}{ Tipo de quimioterápicos orais diferentes } \\
\hline 1 quimioterápico & 31 & 68,8 \\
\hline 2 quimioterápicos & 11 & 24,5 \\
\hline 3 quimioterápicos & 3 & 6,7 \\
\hline
\end{tabular}

Fonte: Autores.

No que tange às características, observou-se o seguinte: $42,3 \%$ crianças do sexo feminino e 57,7\% do sexo masculino, com duas faixas etárias predominantes, de 5 a 11 anos $(37,8 \%)$ e de 12 a 19 anos (53,3\%). Em relação ao tempo do diagnóstico, identificou-se $33,4 \%$ das crianças e adolescentes variando de meses a dois anos e $37,8 \%$, de 2 a 4 anos, sendo a média de dois anos. 
Assim, a maior parte das crianças e adolescentes do estudo encontrava-se na segunda infância (37.8\%) e na adolescência (53,3\%). Esses dados corroboram outras pesquisas realizadas sobre a incidência e a mortalidade por câncer infantojuvenil, demonstrando maior ocorrência entre indivíduos do sexo masculino do que no sexo feminino e os adolescentes de 15 a 19 anos sendo mais acometidos por neoplasias do que crianças compreendidas entre 0 e 14 anos (Monteiro, Fernandes, Soares \& Hyodo, 2018; Mutti et al., 2018).

Quanto ao diagnóstico, verificou-se que 62,3\% das crianças e adolescentes realizavam tratamento para doenças oncohematológicas (sendo o tipo mais predominante as Leucemias, 55,6\%) e 37,7\% crianças e adolescentes em tratamento para tumores sólidos, da oncopediatria. Dessas 17 crianças, 64,7\% possuíam diagnóstico para tumores do sistema nervoso central. Tais achados da pesquisa reforçam os dados do Ministério da Saúde, os quais apontam que, entre os tipos de câncer infantojuvenil, a leucemia é o mais comum na maioria das populações (cerca de $25 \%$ a $35 \%$ ). Os tumores do sistema nervoso central, o mais frequente tumor sólido na faixa etária pediátrica, ocorrem principalmente em crianças menores de 15 anos, com um pico na idade de 10 anos, e os linfomas correspondem ao terceiro tipo de câncer mais comum (INCA, 2016).

No que se refere aos quimioterápicos orais, os mais utilizados foram a Mercaptopurina (29,1\%), o Metrotexato (21\%) e o Imatinibe (19,3\%), indicados para o tratamento de doenças oncohematológicas como as Leucemias, Linfomas e Histiocitose. Outros quimioterápicos orais que tiveram destaque foram a Temozolamida $(9,7 \%)$ e o Everolimus $(8,1 \%)$, utilizados para o tratamento de tumores do sistema nervoso central.

Ao analisar os antineoplásicos orais mais utilizados, observou-se que 62,3\% faziam uso de drogas referentes a tratamentos para doenças oncohematológica e $37,7 \%$ das crianças e adolescentes utilizavam para o tratamento de tumores sólidos e paliativos.

Ainda, constatou-se que 31,2\% das crianças e adolescentes recebiam como tratamento a associação de mais de um quimioterápico oral e 68,8\%, apenas uma droga antineoplásica. Essa condição pode se transformar em um problema para os familiares, pois estes podem apresentar dúvidas na administração dos quimioterápicos pelo protocolo conter drogas diferentes, com horários e doses distintas, esse fator pode dificultar a adesão à terapêutica (Linder et al., 2019).

Estudo apontou que a adesão adequada à terapia de manutenção para a LLA, incluindo a Mercaptopurina diária e o Metotrexato semanal, é essencial para a remissão da doença. E as taxas de não adesão a esses medicamentos entre os pacientes pediátricos podem variar de $27 \%$ a $98 \%$ (Wu et al., 2018). O uso correto dos quimioterápicos orais contribui para melhor resultado do tratamento e aumento nas taxas de sobrevida ao longo dos anos (Pui, Yang, Bhakta \& Rodriguez-Galindo, 2018; Tessmann et al., 2020).

Quanto aos achados dessa pesquisa, foi possível identificar as principais drogas antineoplásicas orais utilizadas pela clientela infantojuvenil e, assim, descrever as principais recomendações para os cuidados domiciliares às crianças e adolescentes que fazem uso das mesmas.

\section{Mercaptopurina}

A Mercaptopurina é indicada para o tratamento das leucemias agudas. Sua apresentação é em comprimidos, com frasco contendo 25 comprimidos de cor branca na dosagem de $50 \mathrm{mg}$. O familiar precisa ser orientado a manter os comprimidos em sua embalagem original e em temperatura ambiente, protegida da luz e da umidade. Faz interação medicamentosa com o Alopurinol, Azatioprina e a Varfarina (Bonassa \& Gato, 2012; ANVISA, 2021; Buzaid, Maluf, \& Lima, 2015).

Quanto às recomendações para a administração, os comprimidos não devem ser partidos, triturados ou mastigados. A criança e o adolescente devem aumentar ingesta hídrica e, em caso de náuseas, o antiemético deve ser administrado 30 minutos antes da Mercaptopurina. Deve-se tomar a Mercaptopurina com o estômago vazio, de duas a quatro horas após as refeições, 
evitar ingestão de alimentos e líquidos após as duas primeiras horas da administração do quimioterápico (Bonassa \& Gato, 2012; ANVISA, 2021; Buzaid et al., 2015; Tessmann et al., 2020).

Estudos sugerem que a administração de Mercaptopurina durante a noite diminui o risco de reincidência em comparação com a administração pela manhã (ANVISA, 2021; Tessmann et al., 2020; Pui et al., 2018; Dreisig et al., 2020). Os principais efeitos colaterais são: mielossupressão (leucopenia, trombocitopenia); gastrintestinais (náuseas, vômito, diarreia e anorexia); cutâneas (rash cutâneo); e fadiga. Exige o controle da função renal e hepática. Não há antídoto conhecido, assim, em casos de acidentes na ingestão maior que o prescrito, o quadro sanguíneo deve ser cuidadosamente monitorado e o familiar, procurar uma emergência para que medidas de suporte adequadas sejam tomadas (Bonassa \& Gato, 2012; ANVISA, 2021; Buzaid et al., 2015; INCA, 2020b; Whiley, Price \& MacDonald, 2020).

\section{Metotrexato}

É indicado para o tratamento de diversos tipos de tumores como as leucemias e os linfomas. Sua apresentação é em comprimidos de 2,5 mg de coloração amarela. Deve ser armazenado em temperatura ambiente, protegido da luz e da umidade, bem fechado em sua embalagem original. Faz interação medicamentosa com a Varfarina, Cetoprofeno, Ibuprofeno, Diclofenaco, Nimesulida, Omeprazol, Pantoprazol, Amoxicilina e a Penicilina (Bonassa \& Gato, 2012; ANVISA, 2021; Buzaid et al., 2015).

Quanto às recomendações para a administração, os comprimidos não devem ser partidos, triturados ou mastigados. O familiar deve incentivar o aumento da ingesta hídrica. Em caso de náuseas, o antiemético deve ser administrado 30 minutos antes do Metotrexato. Evitar exposição solar. É recomendado que o Metotrexato seja administrado em jejum ou com o estomago vazio (Bonassa \& Gato, 2012; ANVISA, 2021; Buzaid et al., 2015; INCA 2020b; Tessmann et al., 2020).

Os principais efeitos colaterais são: mielossupressão (anemia, leucopenia e/ou trombocitopenia); gastrintestinais (náusea, vômito, diarreia, mucosite); e cutâneas (eritema). Exige o controle da função hepática e renal. Em caso de superdose, a leucovorina é indicada para diminuir a toxicidade do Metotrexato e deve ser administrada rapidamente após a superdose. O familiar deve procurar uma emergência para que medidas de suporte adequadas sejam tomadas (Bonassa \& Gato, 2012; ANVISA, 2021; Buzaid et al., 2015; INCA, 2020b; Whiley et al., 2020).

\section{Imatinibe}

Indicado para o tratamento de pacientes pediátricos com Leucemia Mieloide Crônica (LMC) cromossomo Philadelphia positivo $(\mathrm{Ph}+)$; e pacientes adultos e pediátricos com Leucemia Linfoblástica Aguda (LLA) cromossomo Philadelphia positive $(\mathrm{Ph}+)$. Apresentado em comprimidos de $100 \mathrm{mg}$ e $400 \mathrm{mg}$, em frascos/embalagens contendo 30, 60, 200 ou 600 comprimidos revestidos. O quimioterápico deve ser armazenado em temperatura ambiente, protegido da luz e da umidade, bem fechado em sua embalagem original. Faz interação medicamentosa com a Dexametasona, Fenitoína, Carbamazepina, Rifampicina, Fenobarbital e a erva de São João (Bonassa \& Gato, 2012; ANVISA, 2021; Buzaid et al., 2015).

Quanto à administração, os comprimidos não devem ser partidos, triturados ou mastigados. O familiar deve estimular o aumento da ingesta hídrica. Em caso de náuseas, o antiemético deve ser administrado 30 minutos antes do Imatinibe. Recomendada a administração durante uma refeição, com um copo grande de água para minimizar os riscos de distúrbios gastrointestinais. Dependendo da dose, a administração do Imatinibe pode ser dividida em duas - uma pela manhã e outra à noite (Bonassa \& Gato, 2012; ANVISA, 2021; Buzaid et al., 2015; INCA, 2020b; Tessmann et al., 2020; Cruz, Santos, Santos, Trovatti \& Fuly, 2017).

Os efeitos colaterais mais comuns são: mielossupressão (neutropenia, trombocitopenia e anemia); gastrintestinais (anorexia, náusea, vômito, diarreia e dor abdominal); retenção hídrica; edema; e fadiga. Neurológicas (cefaléia, tonturas, 
alteração no paladar); espasmos e câimbras musculares; respiratório (dispneia e tosse); e distúrbios oculares (edema palpebral, hemorragia conjuntival, conjuntivite e visão turva). Em caso de acidente durante a ingestão com uma superdose, como não há antídoto conhecido, o quadro sanguíneo deve ser cuidadosamente monitorado. $\mathrm{O}$ familiar deve procurar uma emergência para que medidas de suporte adequadas sejam tomadas (Bonassa \& Gato, 2012; ANVISA, 2021; Buzaid et al., 2015; INCA, 2020b; Alves, Tavares \& Borges, 2020; Cruz et al., 2017).

\section{Temozolamida}

Indicado para o tratamento de pacientes com glioma maligno recidivante, como o glioblastoma multiforme ou astrocitoma anaplásico. Sua apresentação é em cápsulas, com frascos contendo 5 capsulas de $5 \mathrm{mg}, 10 \mathrm{mg}, 20 \mathrm{mg}, 100 \mathrm{mg}$ e 250mg. As cápsulas possuem cores diferentes de acordo com miligrama. O quimioterápico deve ser armazenado em temperatura ambiente, protegido da luz e da umidade, bem fechado em sua embalagem original. Faz interação medicamentosa com as Quinolonas orais. As cápsulas não devem ser abertas, mastigadas, amassadas ou divididas (Bonassa \& Gato, 2012; ANVISA, 2021; Buzaid et al., 2015).

Quanto às recomendações para a administração, o familiar deve incentivar o aumento da ingestão de líquidos. Em caso de náuseas e vômito, o antiemético deve ser administrado 30 minutos antes da Temozolamida. O familiar deve ser orientado para que as cápsulas sejam engolidas inteiras, acompanhadas de água. A administração pode ser feita em jejum ou uma hora antes das refeições. Se a criança vomitar depois de ter tomado a dose do dia, não deve fornecer uma segunda dose no mesmo dia, pois a Temozolamida é rapidamente absorvida. Caso esqueça de administrar as cápsulas no horário recomendado, o faça assim que lembrar. Mas não dobre a dose se lembrar apenas no dia seguinte (Bonassa \& Gato, 2012; ANVISA, 2021; Buzaid et al., 2015).

Os principais efeitos colaterais são: mielossupressão (neutropenia); gastrintestinais (náuseas e vômitos); neurológicas (cefaleia, sonolência, dificuldade de concentração, tontura, alteração do paladar e parestesia); fadiga; erupção cutânea; prurido; e alopecia. Em caso de acidente com uma dose superior à prescrita, podem ocorrer sintomas relatados em reações adversas de maior intensidade. Nesse caso, o quadro sanguíneo deve ser cuidadosamente monitorado. O familiar deve procurar uma emergência para que medidas de suporte adequadas sejam tomadas (Bonassa \& Gato, 2012; ANVISA, 2021; Buzaid et al., 2015; INCA, 2020b; Alves et al., 2020).

\section{Everolimos}

É indicado para o tratamento de diversos tipos de tumores, como os tumores neuroendócrinos avançados, Angiomiolipoma renal e Astrocitoma subependimário de células gigantes, ambos associados ao Complexo da Esclerose Tuberosa. Sua apresentação é em frascos de $2,5 \mathrm{mg}, 5 \mathrm{mg}$ ou $10 \mathrm{mg}$ contendo 30 comprimidos. Deve ser armazenado em temperatura ambiente, protegido da luz e da umidade, bem fechado em sua embalagem original. Faz interação medicamentosa com antifúngicos, Claritromicina, Fenitoína, Carbamazepina, Fenobarbital, Midazolam, alguns corticosteroides como Dexametasona, Prednisona ou Prednisolona, e a erva de São João. Interações alimentares: com as frutas laranja e carambola ou o suco de laranja (grapefruit). Esses alimentos podem aumentar a quantidade do Everolimos no sangue (Bonassa \& Gato, 2012; ANVISA, 2021; Buzaid et al., 2015; INCA, 2020b).

Em relação às recomendações para a administração, os comprimidos não devem ser partidos, triturados ou mastigados. Incentivar o aumento da ingesta hídrica. Em caso de náuseas, o antiemético deve ser administrado 30 minutos do Everolimos. A administração deve ocorrer todos os dias no mesmo horário regularmente, com ou sem alimentação. A criança precisa engolir os comprimidos inteiros com um copo de água. Caso o familiar esqueça de fornecer o Everolimos à criança, ainda pode fazê-lo até 6 horas após o horário habitual. Mas, se passar de 6 horas, a orientação é que se pule essa dose e, no dia 
seguinte, dê o comprimido no horário prescrito. Nunca deve ser administrada uma dose dobrada para compensar a dose perdida (Bonassa \& Gato, 2012; ANVISA, 2021; Buzaid et al., 2015).

Os efeitos colaterais mais comuns são: sinais de infecção como aumento da temperatura ou calafrios; fadiga; gastrintestinais (anorexia, náusea, diarreia, mucosite, icterícia e dor abdominal); e cefaleia. É necessário o controle da função hepática e renal. É recomendada a atenção do familiar quanto aos sinais de reação alérgica. Em caso de superdosagem, o quadro sanguíneo deve ser cuidadosamente monitorado. O familiar deve procurar uma emergência para que medidas de suporte adequadas sejam tomadas (Bonassa \& Gato, 2012; ANVISA, 2021; Buzaid et al., 2015; INCA, 2020b).

\section{Ciclofosfamida}

A ciclofosfamida é indicada para o tratamento de vários tipos de tumores. Sua apresentação é em comprimidos de 50 $\mathrm{mg}$ na cor branca. $\mathrm{O}$ familiar precisa ser orientado para que os comprimidos permaneçam na embalagem original, na geladeira com temperatura entre $2^{\circ} \mathrm{C} \mathrm{e} 8^{\circ} \mathrm{C}$. Proteger da luz e da umidade. Outro aspecto importante é que faz interação com Alopurinol, Varfarina, Hidroclorotiazida e Clorpromazina, assim, o enfermeiro deve sempre perguntar sobre o uso desses medicamentos (Bonassa \& Gato, 2012; ANVISA, 2021; Buzaid et al., 2015).

Quanto às recomendações para a administração, os comprimidos não devem ser partidos, triturados ou mastigados. A criança deve aumentar ingestão hídrica e esvaziar a bexiga em intervalos regulares. Em caso de náuseas, o antiemético deve ser administrado 30 minutos da Ciclofosfamida. A administração deve ser feita pela manhã e nunca à noite, pois a criança passará várias horas sem ingerir líquidos e urinar. Os principais efeitos colaterais são mielossupressão, náuseas e vômitos, cistite hemorrágica, alopecia e escurecimento da pele e das unhas. É preciso destacar que não há um antídoto específico e, em casos de acidentes na ingestão maior que o prescrito, medidas de suporte adequadas devem ser tomadas; procure uma emergência (Bonassa \& Gato, 2012; ANVISA, 2021; Buzaid et al., 2015; INCA, 2020b).

\section{Tioguanina}

Indicado para o tratamento de certos tipos de leucemia. Sua apresentação é em comprimidos, na dosagem de $40 \mathrm{mg}$. Deve ser armazenado em temperatura ambiente, protegido da luz e da umidade, bem fechado em sua embalagem original. Os comprimidos não devem ser partidos, triturados ou mastigados. O familiar deve estimular o aumento da ingestão hídrica. Em caso de náuseas, o antiemético deve ser administrado 30 minutos da Tioguanina. É recomendada a administração do quimioterápico com o estômago vazio, entre as refeições (para facilitar a absorção), além de evitar a ingestão de alimentos e líquidos após as duas primeiras horas da administração do quimioterápico (os níveis plasmáticos da tioguanina podem ser reduzidos após a ingestão de alimentos) (Bonassa \& Gato, 2012; ANVISA, 2021; Buzaid et al., 2015; Tessmann et al., 2020).

Os efeitos colaterais mais comuns são: mielossupressão (leucopenia e trombocitopenia); gastrintestinais (náuseas, vômito, mucosite e diarreia); hepatotoxidade (controle da função hepática); e rash cutâneo. É provável que a toxicidade hematológica (sanguínea) seja mais intensa com o uso crônico. Em caso de superdose, não há um antídoto específico. O familiar deve procurar uma emergência para que medidas de suporte adequadas sejam tomadas (Bonassa \& Gato, 2012; ANVISA, 2021; Buzaid et al., 2015; INCA, 2020b).

\section{Etoposideo}

É indicado para o tratamento de diversos tipos de tumores. Sua apresentação é em cápsulas gelatinosas na dosagem de $50 \mathrm{mg}$ e $100 \mathrm{mg}$. Deve ser armazenado em temperatura ambiente, protegido da luz e da umidade, bem fechado em sua embalagem original. Faz interação medicamentosa com Aprepitanto, Varfarina e erva de São João (Bonassa \& Gato, 2012; ANVISA, 2021; Buzaid et al., 2015). 
Quanto às recomendações para a administração, as cápsulas não devem ser abertas, mastigadas, amassadas ou divididas. O familiar deve incentivar o aumento da ingestão de líquidos. Em caso de náuseas e vômito, o antiemético deve ser ministrado 30 minutos antes da administração do Etoposideo. O quimioterápico deve ser administrado com o estômago vazio (Bonassa \& Gato, 2012; ANVISA, 2021; Buzaid et al., 2015).

Os efeitos colaterais mais comuns são: mielossupressão (anemia, leucopenia); gastrintestinais (náuseas e vômitos, anorexia, estomatite e diarreia); e cutâneas (alopecia e reações alérgicas como prurido e erupção cutânea). Em acidente com uma superdose, não há um antídoto específico. O familiar deve procurar uma emergência para que medidas de suporte adequadas sejam tomadas (Bonassa \& Gato, 2012; ANVISA, 2021; Buzaid et al., 2015; INCA, 2020b).

\section{Topotecano}

É indicado para o tratamento de pacientes com recidiva de câncer de pulmão de pequenas células. A apresentação é em frasco contendo 10 cápsulas de 0,25 mg e de $1 \mathrm{mg}$. Quanto ao armazenamento, deve ser mantido em sua embalagem original, sob refrigeração em temperatura entre $2^{\circ} \mathrm{C}$ e $8^{\circ} \mathrm{C}$ e protegido da luz; não deve ser congelado. Faz interação medicamentosa com alguns quimioterápicos como a Cisplatina, Carboplatina, Paclitaxel e o Etoposideo (Bonassa \& Gato, 2012; ANVISA, 2021; Buzaid et al., 2015).

Quanto às recomendações para a administração, as cápsulas não devem ser abertas, mastigadas, amassadas ou divididas. O familiar deve incentivar a criança e o adolescente a aumentar a ingestão de líquidos. Em caso de náuseas e vômito, o antiemético deve ser administrado 30 minutos antes do Topotecano. Caso o familiar esqueça de fornecer o quimioterápico, não é indicada dose duplicada para repor a esquecida. Deve-se apenas seguir com o tratamento, ingerindo normalmente o Topotecano no dia seguinte. Pode ser administrado com ou sem alimentos (Bonassa \& Gato, 2012; ANVISA, 2021; Buzaid et al., 2015).

Os principais efeitos colaterais são: mielossupressão (neutropenia, trombocitopenia e anemia); gastrintestinais (náusea, vômito, diarreia, dor abdominal e anorexia); fadiga; e alopecia parcial. Em caso de superdose, os sinais e sintomas são correspondentes às reações adversas. Visto que não há antidoto conhecido, o quadro sanguíneo deve ser cuidadosamente monitorado. $\mathrm{O}$ familiar deve procurar uma emergência para que medidas de suporte adequadas sejam tomadas (Bonassa \& Gato, 2012; ANVISA, 2021; Buzaid et al., 2015; INCA, 2020b).

\section{Pazopanibe}

Indicado para o tratamento de carcinoma de células renais avançado e/ou metastáticos e para o tratamento de pacientes com sarcoma de partes moles avançado, que receberam quimioterapia prévia. A apresentação é em comprimidos revestidos de $200 \mathrm{mg}$ ou $400 \mathrm{mg}$. Deve ser armazenado em temperatura ambiente, protegido da luz e da umidade, bem fechado em sua embalagem original. Faz interação medicamentosa com a Amiodarona, Azitromicina, Fluconazol, Ondansetrona e a Sinvastatina (Bonassa \& Gato, 2012; ANVISA, 2021; Buzaid et al., 2015).

Quanto às recomendações para a administração, os comprimidos não devem ser partidos, triturados ou mastigados. Aumentar ingestão hídrica. Em caso de náuseas, o antiemético deve ser ministrado 30 minutos do Pazopanibe. Deve ser administrado preferencialmente em jejum ou sem alimentos (pelo menos uma hora antes ou duas horas após uma refeição). Caso o familiar esqueça de administrar uma dose, não é indicado fazê-lo antes de um intervalo de 12 horas da próxima dose e não se deve tomar dose duplicada para repor a esquecida. Apenas siga com o tratamento normalmente no dia seguinte como foi prescrito (Bonassa \& Gato, 2012; ANVISA, 2021; Buzaid et al., 2015; INCA, 2020b).

Os principais efeitos colaterais são: gastrintestinais (anorexia, diarreia, náuseas, vômito, dor abdominal e aumento de proteínas do fígado); cefaleia; alteração na cor dos cabelos e pelos; cansaço e fadiga; e cardíaca (bradicardia assintomática). 
Em caso de ingestão acidental ou intencional de grande quantidade do Pazopanibe, o familiar deve procurar uma emergência para que medidas de suporte adequadas sejam tomadas (Bonassa \& Gato, 2012; ANVISA, 2021; Buzaid et al., 2015; INCA, 2020b; Alves et al., 2020).

\section{Considerações Finais}

Este estudo possibilitou identificação dos quimioterápicos antineoplásicos orais utilizados em crianças e adolescentes, abordando as principais orientações para os cuidados domiciliares. $O$ enfermeiro, que tem um importante papel na assistência à criança e ao adolescente em terapia com antineoplásicos orais, pode utilizar essas informações durante a consulta de enfermagem com a finalidade de direcionar ações de orientação quanto à terapia visando à adesão e maior segurança no tratamento.

Os resultados obtidos neste estudo, podem contribuir para a construção de instrumentos utilizados por enfermeiros para a prática de orientação dos familiares de crianças e adolescentes em tratamento com antineoplásicos orais, adequando-se as diferentes realidades sociais, linguagem e rotinas institucionais.

Como limitação deste estudo, pontua-se que os resultados representam a realidade cotidiana de apenas uma instituição, o que pode não retratar a totalidade de pessoas que vivenciam este universo.

\section{Referências}

Agência Nacional de Vigilância Sanitária. Consultas Bulário Eletrônico. (2021). Recuperado de http://consultas.anvisa.gov.br/\#/bulário/

Alves, E. A., Tavares, G. G., Borges, L. L. (2020). Importância da atenção farmacêutica para a quimioterapia antitumoral. Revista Brasileira Militar de Ciências, 6(15).

Bonassa, E. M. A., \& Gato, M. I. R. (2012). Terapêutica oncológica para enfermeiros e farmacêuticos. Rio de Janeiro: Atheneu.

Cruz, P. S., Santos, M. L. S. C dos, Santos, W. A, Trovatti, P., \& Fuly, P. S. C. (2017). Qualidade de vida dos pacientes com leucemia mieloide crônica em uso de Imatinibe. Revista de Enfermagem UFPE, 11(6), 2423-2431.

Dreisig, K., Brünner, E. D., Marquart, H. V., Helt, L. R., Nersting, J., Frandsen, T. L., Jonsson, O. G., Taskinen, M., Vaitkeviciene, G., Lund, B., Abrahamsson, J., Lepik, K., \& Schmiegelow, K. (2020). TPMT polymorphisms and minimal residual disease after 6-mercaptopurine post-remission consolidation therapy of childhood acute lymphoblastic leukaemia. Pediatric Hematology and Oncology, 1-12.

Fernandes, A. F. F, Silva, S. S., Tacla, M. T. G. M, Ferrari, R. A. P, \& Gabani, F. L. (2018). Informações aos pais: um subsídio ao enfrentamento do câncer infantil. Semina: Ciências Biológicas e da Saúde, 39(2), 145-152.

Instituto Nacional de Câncer José Alencar Gomes da Silva. (2016). Coordenação de Prevenção e Vigilância. Incidência, mortalidade e morbidade hospitalar por câncer em crianças, adolescentes e adultos jovens no Brasil: informações dos registros de câncer e do sistema de mortalidade. Rio de Janeiro: Ministério da Saúde. Recuperado de http://www1.inca.gov.br/inca/Arquivos/estimativa-2020.pdf

Instituto Nacional de Câncer Jose Alencar Gomes da Silva. (2020a). Estimativa 2020: Incidência de câncer no Brasil. Rio de Janeiro: Ministério da Saúde. Recuperado de http://www1.inca.gov.br/inca/Arquivos/estimativa-2020.pdf

Instituto Nacional de Câncer José Alencar Gomes da Silva. (2020b). Quimioterapia: orientações aos pacientes. Rio de Janeiro: Ministério da Saúde. Recuperado de http://www1.inca.gov.br/inca/publicacoes/cartilhas/quimioterapia

Iuchno, C. W, \& Carvalho, G. P. (2019). Toxicidade e efeitos adversos decorrente do tratamento quimioterápico antineoplásico em pacientes pediátricos: revisão integrativa. Ciência \& Saúde, 12(1), e30329.

Linder, L. A., Wu, Y. P., Macpherson, C. F., Fowler, B., Wilson, A., Jo, Y., Jung, S. H., Parsons, B., \& Johnson, R. (2019). Oral medication adherence among adolescents and young adults with cancer before and following use of a smartphone-based medication reminder App. Journal of Adolescent and Young Adult Oncology, 8(2), 122-130.

Buzaid, A. C., Maluf, F. C., \& Lima, C. M. R. Manual de Oncologia Clínica do Brasil. Agentes Oncológicos. (2015). São Paulo: Dendrix.

Marshall, V. K, \& Cairns, P. L. (2019). Challenges of caregivers of cancer patients who are on oral oncolytic therapy. Seminars in Oncology Nursing, 35, 363369.

Mesquita, M. E. R., \& Silva, R. P. (2016). Autocuidado e quimioterapia oral domiciliar: avaliação das práticas educativas dos enfermeiros sob a perspectiva de pacientes. Revista Brasileira de Cancerologia, 62(3), 237-245. 
Research, Society and Development, v. 10, n. 4, e40310414530, 2021

(CC BY 4.0) | ISSN 2525-3409 | DOI: http://dx.doi.org/10.33448/rsd-v10i4.14530

Monteiro, N. M. L., Fernandes, F. L., Soares, I. A. F., \& Hyodo, L. T. M. M. C. (2018). Perfil clínico e epidemiológico dos pacientes de um serviço de oncologia pediátrica de um hospital do leste de Minas Gerais. Revista Médica de Minas Gerais, 28(e-1961), 1-7.

Mutti, C. F., Cruz, V. G., Santos, L. F., Araújo, D., Cogo, S. B., \& Neves, E. T. (2018). Perfil clínico-epidemiológico de crianças e adolescentes com câncer em um serviço de oncologia. Revista Brasileira de Cancerologia; 64(3), 293-300.

Pui, C. H., Yang, J. J., Bhakta, N., \& Rodriguez-Galindo, C. (2018). Global efforts toward the cure of childhood acute lymphoblastic leukaemia. Lancet Child Adolesc Health, 2(6), 440-454.

Tessmann, L., Medeiros-Souza, P., Cordoba, J. C. M., Tavares, N. U. L., Abílio, V. M., Matos, D. O., \& Magalhães, I. M. Q. S. (2020). Partição de Comprimidos Antineoplásicos Utilizados no Tratamento de Leucemias Agudas em Crianças e Adolescentes. Revista Brasileira de Cancerologia, 66(2), e01764.

Whiley, A. C., Price, V., \& MacDonald, T. (2020). An exploration of mercaptopurine/methotrexate tolerance during maintenance therapy in children with acute lymphoblastic leukemia. Journal of Oncology Pharmacy Practice, 1-6.

Wu, Y. P., Stenehjem, D. D., Linder, L. A., Yu, B., Parsons, B. G., Mooney, R., \& Fluchel, M. N. (2018). Adherence to oral medications during maintenance therapy among children and adolescents with Acute Lymphoblastic Leukemia: a medication refill analysis. Journal of Pediatric Oncology Nursing, 35(2), 8693. 